Canadian

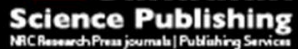

Canadian Journal of Physiology and Pharmacology Revue canadienne de physiologie et pharmacologie

\title{
Infliximab alleviates the mortality, mesenteric hypoperfusion, aortic dysfunction and multiple organ damage in septic rats
}

\begin{tabular}{|r|l|}
\hline Journal: & Canadian Journal of Physiology and Pharmacology \\
\hline Manuscript ID & cjpp-2016-0628.R1 \\
\hline Manuscript Type: & Article \\
\hline Date Submitted by the Author: & 25 -Jan-2017 \\
\hline Complete List of Authors: & $\begin{array}{l}\text { Ozer, Erdem; Selcuk University, Faculty of Medicine, Pharmacology } \\
\text { Goktas, Mustafa; Yildirim Beyazit University Faculty of Medicine, } \\
\text { Pharmacology } \\
\text { Kilinc, Ibrahim; Necmettin Erbakan University Faculty of Medicine, } \\
\text { Biochemistry } \\
\text { Toker, Aysun; Necmettin Erbakan University Faculty of Medicine, } \\
\text { Biochemistry } \\
\text { Bariskaner, Hulagu ; Selcuk University Faculty of Medicine, Pharmacology } \\
\text { Ugurluoglu, Ceyhan; Selcuk University Faculty of Medicine, Pathology } \\
\text { Iskit, Alper; Hacettepe University, Faculty of Medicine, Pharmacology }\end{array}$ \\
\hline Keyword: & $\begin{array}{l}\text { infliximab, sepsis, survival, multiple organ dysfunction, vascular reactivity } \\
\text { and mesenteric arterial blood flow }\end{array}$ \\
\hline
\end{tabular}


Infliximab alleviates the mortality, mesenteric hypoperfusion, aortic dysfunction and multiple organ damage in septic rats

Erdem Kamil Ozer ${ }^{1}$, Mustafa Tugrul Goktas ${ }^{2}$, Ibrahim Kilinc ${ }^{3}$, Aysun Toker ${ }^{3}$, Hulagu Bariskaner ${ }^{1}$, Ceyhan Ugurluoglu ${ }^{4}$, Alper Bektas Iskit ${ }^{5}$

1 Department of Pharmacology, Faculty of Medicine, Selcuk University, Konya, Turkey

2 Department of Pharmacology, Faculty of Medicine, Yildirim Beyazit University, Ankara, Turkey

3 Department of Biochemistry, Faculty of Medicine, Necmettin Erbakan University, Konya, Turkey

4 Department of Pathology, Faculty of Medicine, Selcuk University, Konya, Turkey

5 Department of Pharmacology, Faculty of Medicine, Hacettepe University, Ankara, Turkey

Corresponding author:

Erdem Kamil OZER, MD

Present Address: Selcuk University, Faculty of Medicine, Department of Pharmacology,

Selcuklu, Konya, Turkey, 42250.

Phone: +90 $3322243839 \quad$ Fax: +90 3322412184

E-mail: drekfarma@hotmail.com 


\begin{abstract}
Tumor necrosis factor-alpha (TNF- $\alpha$ ) is a pivotal mediator that triggers inflammatory process, oxidative stress and multiple organ injury in sepsis. We investigated the effects of infliximab on survival, mesenteric artery blood flow (MBF), vascular reactivity, oxidative and inflammatory injuries in cecal ligation and puncture (CLP) induced sepsis. Wistar rats were divided into Sham, CLP, Sham+infliximab, CLP+infliximab subgroups. 24 hours before the operations rats were injected intraperitoneally with infliximab $(7 \mathrm{mg} / \mathrm{kg})$ or vehicle (saline; 1 $\mathrm{mL} / \mathrm{kg}$ ). 20 hours after operations MBF and phenylephrine responses of isolated aortic rings were measured. Tissue damages were examined biochemically and histopathologically. Furthermore, survival rates were monitored throughout 96 hours. Infliximab improved survival, mesenteric perfusion and aortic function after CLP. Increases of serum AST, ALT, LDH, BUN, Cr and inflammatory cytokines (tumor necrosis factor-alpha, interleukin-1 beta and interleukin-6) induced by CLP were blocked by infliximab. Infliximab prevented malondialdehyde elevations in septic liver, lung, spleen and kidney tissues and as well as glutathione reductions in septic liver, spleen and kidney tissues. Protective effects of infliximab on multiple organ damage were also observed histopathologically. Infliximab showed protective effects in sepsis due to its improvement effects on mesenteric perfusion, aortic function and its anti-inflammatory and antioxidative effects.
\end{abstract}

Keywords: Infliximab, sepsis, survival, multiple organ dysfunction, vascular reactivity and mesenteric arterial blood flow 


\section{Introduction}

Although all improved and labored treatment strategies to remedy of sepsis, it is still major cause of mortality and morbidity especially in the intensive care units with very high cost of treatment (Dellinger et al. 2013; Marik 2011). In the past decades, major advances have been made in understanding the process and pathological mechanism of sepsis. In early septic process, invasion of microorganisms into the bloodstream following mesenteric ischaemia leads to massive and uncontrolled release of the proinflammatory cytokines, especially tumor necrosis factor-alpha (TNF- $\alpha$ ) and interleukin-1 beta (IL-1 $\beta$ ), and free oxygen radicals (De Backer et al. 2014; Iskander et al. 2013; Yuksel et al. 2009).

TNF- $\alpha$, the key mediator in sepsis, triggers the cascade of pathophysiological events in septic process including overproduction of the other cytokines such as IL-1 $\beta$ and IL-6, mitochondrial dysfunction, hypoxia, oxidative stress, intravascular coagulation, vasoplegia, multisystem hypoperfusion, severe sepsis (life-threatening organ dysfunction caused by a dysregulated host response to infection), septic shock (vasopressor requirement to maintain a mean arterial pressure of $65 \mathrm{~mm} \mathrm{Hg}$ or greater and serum lactate level greater than $2 \mathrm{mmol} / \mathrm{L}$ in the absence of hypovolemia), multiple organ dysfunction syndrome (MODS) and even death (Duran-Bedolla et al. 2014; Ferro et al. 2000; Ley et al. 2007; Schulte et al. 2013; Singer et al. 2016). It was shown that TNF- $\alpha$ inhibition has beneficial effects on the survival of Staphylococcal enterotoxin-induced septic shock in a mouse model (Fei et al. 2011). Infliximab is a human-murine chimeric monoclonal antibody against TNF- $\alpha$ that was approved for the treatment of autoimmune diseases such as Crohn's disease, ulcerative colitis, ankylosing spondylitis and rheumatoid arthritis (Lis et al. 2014). It has also been reported that infliximab shows anti-inflammatory and antioxidant effects in a rat model of intestinal ischemia/reperfusion injury (Pergel et al. 2012).

In the light of these previous findings, we aimed to investigate the possible beneficial effects of infliximab pretreatment as a preventive strategy in an experimental model of sepsis induced by caecal ligation and puncture (CLP) with particular attention to the decreases in survival, mesenteric blood flow, contractile response of the aortic muscle to phenylephrine and as well as histopathological and biochemical injury in target vital organs such as liver, lung, kidney and spleen. 


\section{Materials and methods}

\section{Animals}

Fifty tree female Wistar albino rats, weighing between 250-300 g, were obtained from the animal shelter of Hacettepe University, Ankara, Turkey. The rats were kept at 30-70\% relative humidity, and $21 \pm 2{ }^{\circ} \mathrm{C}$ temperature, and 12-h light/12-h dark illumination sequence under environmentally controlled conditions and given ad libitum access to tap water (drinking bottle) and standard pellet dairy chow. This study was performed according to The Recommendations from the Declaration of Helsinki together with The Guiding Principles in the Care and Use of Laboratory Animals. Before the commencement of any intervention this study was approved by the Animal Care Committee at Hacettepe University Faculty of Medicine (Approval Number: 2015/101).

\section{Experimental protocols}

Animals were randomly allocated into four groups as Sham (saline as the vehicle of infliximab administered and Sham operated rats), CLP (saline administered and CLP operated rats; septic rats), Sham+infliximab (infliximab administered and Sham operated rats) and CLP+infliximab (infliximab administered and CLP operated rats). Rats were administered with infliximab (Remicade ${ }^{\circledR}, 7 \mathrm{mg} / \mathrm{kg}$ i.p.) or vehicle [nonpyrogenic sterile saline $(0.9 \%$ $\mathrm{NaCl}, w / v$ )] 24 hours before the Sham and CLP operations (Altintas et al. 2016; Saritemur et al. 2015). All animals were fasted overnight but allowed ad libitum access to drinking water one day before the operations.

Twenty-nine animals were observed at 6-h intervals until 96 hours after the Sham and CLP operations for survival assessment. Twenty-four rats were used for mesenteric artery blood flow (MBF) and isometric measurements, histopathological and biochemical analyses. MBF was measured with a Doppler ultrasound flowmeter twenty hours after the Sham and CLP operations. Blood samples were collected via cardiac puncture and transferred to Eppendorf tubes after the MBF measurements. After waiting 15-20 minutes serum was separated by centrifugation at $4000 \mathrm{xg}$ for 5 minutes. Serum samples aliquots were stored at $80^{\circ} \mathrm{C}$ until the assays were performed. Immediately after the collection of the blood samples, tissue samples of liver, lung, kidney and spleen were removed for biochemical and histopathological analyses and aorta was quickly removed for isometric measurements. The pieces of tissue sections were transferred to Eppendorf tubes and stored at $-80^{\circ} \mathrm{C}$ until the assays were performed for measurements of malondialdehyde (MDA) and glutathione (GSH) 
levels. In addition the other pieces of liver, lung, spleen and kidney tissues were harvested accordingly, fixed in 10\% neutral buffered formaldehyde solution for histopathological analyses.

\section{Polymicrobial sepsis model}

Polymicrobial sepsis was induced by CLP operation as described previously (Hubbard et al. 2005; Ozer and Iskit 2016). Briefly, rats were fasted overnight, but were allowed ad libitum access to drinking water one day before the operations. For the operation, the animals were anesthetized with chloral hydrate $(400 \mathrm{mg} / \mathrm{kg}$, i.p.) and a 2-cm ventral midline abdominal incision was performed. The cecum was then exposed, and ligated just below the ileocecal valve and punctured twice with an 18-gauge needle. The punctured cecum was gently massaged in order to expel a small amount of bowel feces and then abdominal incision was closed in two layers by using atraumatic 3-0 silk sutures. After the operation, normal saline ( $3 \mathrm{~mL} / 100 \mathrm{~g}$, subcutaneously) was injected immediately in rat's nape of the neck. For Sham operation all of the same steps were performed, except for cecum ligation and puncture. The rats that died during the surgery and within 20 hours after the Sham and CLP operations were excluded from the study. Several hours after CLP the animals showed clear symptoms of the disease, such as tachypnea, tachycardia, hypotension, hypothermia, piloerection, lethargy, hunched posture, diarrhea, anorexia, and behavioral changes.

\section{Mesenteric artery blood flow measurement}

After twenty hours the Sham and CLP operations rats were anesthetized with chloral hydrate and abdominal incision was performed again. The superior mesenteric artery was then carefully exposed and a perivascular Doppler ultrasonic probe was located around the artery in order to measure the blood flow. The Doppler flow probe was connected to a Transonic Small Animal Flowmeter System T206 (Transonic Inc., Ithaca, N.Y., USA), which provides blood flow readings $(\mathrm{mL} / \mathrm{min})$ absolutely. The rats were left to stabilize for 15 minutes before recording MBF values. The obtained signals from the flowmeter were also recorded on a computer by using a MP35 Biopac data recording system (Goleta, Calif., USA) (Yuksel et al. 2009).

\section{Isometric measurements}

The changes in alpha-receptor mediated vasoconstriction induced by CLP were evaluated on isolated rat aorta preparations by cumulative phenylephrine administration 
which is an $\alpha_{1}$-receptor agonist into the organ bath. Thoracotomy was performed and thoracic part of aorta was then removed and put into the cold Krebs Henseleit $(\mathrm{K}-\mathrm{H})$ solution (as mM; sodium chloride 118 , sodium bicarbonate 25 , potassium chloride 4.7 , calcium chloride 2 , magnesium sulfate 1.2, monopotassium dihydrogen phosphate 1.2, and glucose 10) that gassed with carbogen $\left(95 \% \mathrm{O}_{2} / 5 \% \mathrm{CO}_{2}\right)$. The isolated aorta was cautiously cleaned of surrounding fat and connective tissues and then $3 \mathrm{~mm}$ wide rings were obtained. The isolated aortic rings were mounted on two triangular stainless-steel wire specimen holders and transferred into the $20 \mathrm{~mL}$ isolated organ baths containing containing K-H solution that were kept at $37^{\circ} \mathrm{C}$ and aerated with carbogen. The aortic rings were adjusted to $\sim 1 \mathrm{~g}$ resting tension and allowed to equilibrate in K-H solution for about 45 minutes with washing out of the tissues every 15 minutes (Ozer et al. 2015). The muscle contractions were recorded using a force-displacement transducer and digitized data acquisition system (MP35 from BIOPAC, Goleta, California, USA). After this period phenylephrine $\left(10^{-9}\right.$ to $3 \times 10^{-5}$ Molar [M] $)$ were applied into the organ baths with a cumulative concentration to study receptor mediated constriction. For each phenylephrine concentration, the increase in recorded contraction amplitude changes were expressed as grams of tension (Kalea et al. 2010; Kawabe et al. 2003). The obtained data were fit into a curve, and then EC50 value which is an indicator of potency and Emax value which is an indicator of efficacy were determined by using GraphPad Prism 5 software (GraphPad, UK).

\section{Histopathological examination}

The fixed tissue samples in $10 \%$ buffered formaldehyde solution were embedded in paraffin wax, sectioned at $5 \mu \mathrm{m}$ thickness, mounted on slides, rehydrated, and then stained with hematoxylin and eosin (H\&E) using standard methods. The blinded pathologist examined the tissue sections by the conventional Carl Zeiss Axio Lab.A1 light microscope and photographed majority of them using Axiocam MRc5 microscope camera.

\section{Biochemical parameters}

Before serum analysis, the frozen aliquots of serum samples were thawed and then mixed well. Serum levels of blood urea nitrogen $(\mathrm{BUN})$ and creatinine $(\mathrm{Cr})$, activities of lactate dehydrogenase (LDH), aspartate aminotransferase (AST) and alanine aminotransferase (ALT) were measured by using standard auto-analyzer methods on Abbott Architect 16000 system with the original reagents according to the manufacturer's instructions (Abbott Laboratories, Abbott Park, IL, USA). Hepatic dysfunction was evaluated by measurement the 
increase of serum ALT (a specific marker for hepatic parenchymal injury) and serum AST (a nonspecific marker for liver damage). The levels of BUN and $\mathrm{Cr}$ (an indicator of reduced glomerular filtration rate and hence renal failure) were measured for the renal injury evaluation. LDH levels were measured for tissue damage, although not organ spesific.

As inflammatory markers, serum TNF-alpha (BMS622, eBioscience), IL-1beta (BMS630, eBioscience, San Diego, CA) and IL-6 (BMS625, eBioscience) levels were measured by using sandwich ELISA kits that were specifically designed for rat cytokines, and all measurements were made according to the manufacturer's instructions. Assays of each rat and its correlated control assay were run in the same lot.

MDA levels in liver, spleen, lung and renal tissues were assayed for evaluation of the oxidative damage by using a Lipid Peroxidation Assay Kit (Cat. No. MAK085, SigmaAldrich Co.) according to the manufacturer's protocols. Lipid peroxidation assay is based on evaluation of the MDA level, that is an end product of the lipid peroxidation chain. Thiobarbituric acid (TBA) reacts with MDA to create a colorimetric product. A standard curve was detected by using standard solution of prepared MDA to evaluate the tissue MDA levels. Briefly, MDA Lysis Buffer containing butylhydroxytoluene (BHT) were mixed with each tissue sample. The obtained supernatants were transferred into the glass tubes and reacted with the sodium acetate solution which includes TBA for 60 minutes, at $95^{\circ} \mathrm{C}$. After the centrifugation, the supernatants were collected and the resulting TBA-reactive substances were evaluated at $532 \mathrm{~nm}$ absorbance spectrophotometrically. MDA concentration in each sample was expressed as in $\mathrm{nmol} / \mathrm{mg}$ tissue protein concentration for determination of the lipid peroxidation. For antioxidant status total GSH levels (GSH+GSSG) in each tissue samples were measured using a NADPH linked enzymatic colorimetric glutathione assay kit (Cat. No. CS0260, Sigma-Aldrich Co.) at $412 \mathrm{~nm}$ absorbance and expressed as nmol per mg tissue protein concentration. In short, tissue samples were dissolved within 10 minutes in 5\% 5-Sulfosalicylic Acid (SSA) on ice, and then centrifuged at 10000xg for 10 minutes. The obtained supernatants analyzed according to manufacturer's protocol and the GSH levels in the tissue samples were expressed as nmol per mg tissue protein concentration.

\section{Drugs}

Remicade (Schering-Plough Company); chloral hydrate (Sigma-aldrich); hematoxylin, eosin, formaldehyde, paraffin (Merck).

\section{Statistical analyses}


All data were presented as mean \pm standard deviation (mean \pm SD) and analyzed with the GraphPad Prism 5 programme. All tests that were performed for statistical analyses were shown in the table and figure legends. The differences were considered to be significant when $p<0.05$. Some of the test parameters could not be evaluated in all rats because of the experimental procedure that were mentioned above. So, the " $n$ " values for each subsections were separately given in the table and figure legends for different test parameters. 


\section{Results}

\section{Survival rates}

96-hour survival rates are shown in Fig.1. Within 43 hours after the CLP operation all septic rats in the CLP group died ( $p<0.001$, vs. Sham group). With the administration of infliximab, the survival rate in the CLP+infliximab group increased to $57 \%$ at the end of 96 hours $(p<0.001$ compared to CLP group). Infliximab partially prevented the decrease in survival rate induced by CLP ( $p<0.05$, CLP+infliximab vs. Sham).

\section{Mesenteric artery blood flow (MBF)}

MBF values obtained from the anesthetized rats are shown in Fig.2. The MBF decreased in septic rats from $18.43 \pm 4.86$ (Sham group) to $4.86 \pm 2.03$ (CLP group). However, the infliximab administered animals showed significantly higher MBF values in CLP+infliximab $(15.80 \pm 5.10, p<0.001$ compared to CLP) group. Infliximab ameliorated the CLP induced mesenteric artery dysfunction in septic rats.

\section{Contractile response of aortic smooth muscle}

CLP induced marked hyporesponsiveness to phenylephrine when compared with Sham group $(p<0.001)$ (Fig.3), as shown by reductions in both pD2 (-logEC50 [M], potency) and Emax values (maximal contractile effect, efficacy) for phenylephrine-induced contractions (Table 1). As shown in Table 1, the infliximab pretreatment caused a greater increase in the phenylephrine potency of aortic rings in CLP+infliximab group $(p<0.001$ compared to CLP and $p<0.01$ compared to Sham). However, infliximab partially prevented the CLP-induced reduction in the maximal contractile effect to phenylephrine $(p<0.05$ compared to CLP and $p<0.05$ compared to Sham).

\section{Biochemical results}

The increases in the levels of serum AST, ALT, LDH, BUN and Cr, as well as TNF- $\alpha$, IL-1 $\beta$ and IL-6 induced by CLP were totally prevented by infliximab (Table 2 and 3).

As shown in Table 4, the MDA levels (an indicator for oxidative damage) in the liver, lung, kidney and spleen tissues were significantly higher in the CLP group when compared with the Sham group $(p<0.001)$. Treatment with infliximab totally prevented the CLP-induced elevations of MDA levels in the liver, lung, kidney and spleen tissues. Moreover, CLP caused a significant decrease in GSH levels (an indicator for anti-oxidant status) in all tissues, 
compared to the Sham group $(p<0.05-p<0.001)$. Infliximab prevented the CLP-induced reductions of GSH levels in liver and spleen, but failed to prevent the GSH reductions in the septic lung tissues. Additionally, infliximab partially prevented the kidney GSH reduction induced by CLP.

\section{Histopathological Examinations}

From the histopathological point of view, the structures of liver, lung, spleen and kidney samples were normal in the Sham and Sham+infliximab groups. However, CLP induced substantial histopathological alterations in these samples. In the septic liver tissues, there were moderate sinusoidal portal hemorrhage and mild inflammatory mononuclear cell infiltration in the parenchyma and periportal area (Fig.4-1C). The septic lung tissues showed moderate intra-alveolar hemorrhage, hemosiderin-laden macrophages, mild interstitial and alveolar edema, as well as moderate peribronchial and interstitial inflammation that was concentrated near the vessel wall (Fig.4-2C). The septic spleen tissues showed moderate hemorrhage, severe inflammation and also hemosiderin-laden macrophages (Fig.4-3C). The moderate hemorrhage were observed in the septic kidney parenchyma (Fig.4-4C). When the rats were treated with infliximab the lung (Fig.4-2D) and spleen (Fig.4-3D) architectures were greately improved and as well as liver (Fig.4-1D) and kidney (Fig.4-4D) tissues were completely prevented from the histopathological injuries induced by CLP. 


\section{Discussion}

In early sepsis, mesenteric perfusion is primarily impaired. Decrease of mesenteric blood flow leads to impaired intestinal barrier integrity and therefore translocation of microorganisms into the bloodstream. Spread of the microorganisms triggers massive and uncontrolled release of inflammatory cytokines and subsequent free radicals which ultimately causes negative effects on the vessels and multiple organs (Balzan et al. 2007; Erdem et al. 2007; Koh et al. 2002; Yuksel et al. 2009).

TNF- $\alpha$ is one of the first cytokines to be released in sepsis which amplifies the inflammatory response and oxidative stress (Qiu et al. 2011). TNF- $\alpha$ also induces a vicious cycle promoting mesenteric hypoperfusion, vasoplegia and multiple organ dysfunction (Shimaoka and Park 2008). Therefore, TNF- $\alpha$ inhibition may be an important and approachable therapeutic target for breaking the sepsis induced viscious cycle.

Infliximab is a TNF- $\alpha$ inhibitor which binds with high affinity to both the soluble and the transmembrane forms of TNF- $\alpha$ (Scallon et al. 2002). Infliximab showed antiinflammatory and antioxidant effects in a rat model of intestinal ischemia/reperfusion injury and chronic inflammatory joint diseases such as ankylosing spondylitis and rheumatoid arthritis (Pergel et al. 2012; Tunez et al. 2007). Infliximab also showed benefical effects in an experimental model of endotoxin-induced uveitis (Yuksel et al. 2014). Soluble TNFR II/IgG1 Fc fusion protein, a recombinant fusion protein that consists of the soluble TNF receptor (p75) linked to the Fc portion of human IgG1 and a potent TNF- $\alpha$ inhibitor, protected against sepsis induced hypotension and death when administered 24 hours before endotoxin (lipopolysaccharide, LPS) challenge (Guo et al. 2009). Etanercept binds only to the trimer form of soluble TNF- $\alpha$ that is another TNF- $\alpha$ inhibitor, also improved the survival in a LPSinduced sepsis model (Lee et al. 2013). These promising preclinical effects of TNF- $\alpha$ inhibitors have been shown especially in the endotoxemic rats. However, CLP is more reflective of the clinical reality for sepsis than previous animal models, such as injection of endotoxin or even purified bacteria into the rodents (Raven 2012). Therefore, CLP model was choosen as an experimental sepsis model in this study. To the best of our knowledge, this is the first study that evaluates the possible benefical effects of infliximab on survival, mesenteric blood flow, contractile function of aorta, multiple organ injury after CLP.

In our study, we found that the infliximab administration decreased the mortality, attenuated the mesenteric hypoperfusion, prevented the vascular hyporesponsiveness in the isolated aortic rings, reduced plasma levels of biochemical parameters and as well as 
ameliorated histopathological changes in CLP-induced septic rats. This study provides novel evidence that pretreatment with infliximab seems to improve the survival in the CLP-induced septic rats as a consequence of reduced dysfunction/injury of multiple organs and vessels. These protective effects could be due to attenuation of TNF- $\alpha$ (mainly), IL-1 $\beta$ and IL-6 productions, suppression of oxidative stress, prevention of the mesenteric hypoperfusion and vasoplegia by infliximab. Most of the therapeutic effects of infliximab in septic animals were firstly shown in this study. According to our results some patient groups such as patients with immunosuppression, humans in extremes of age (neonates or elderly) have increased risk for the development of sepsis and infliximab may be given for prophylactic agent.

\section{Conclusion}

In conclusion, our results show that infliximab has protective effect against sepsisinduced mortality and MODS due to improvements of mesenteric hypoperfusion and contractile dysfunction of aorta and its antiinflammatory and antioxidative effects in septic rats. This study is primarily focused on demonstrating the beneficial and protective effects of ant-TNF therapy on prior to sepsis via using a septic animal model. Further clinical trials are also required to demonstrate beneficial effects on early phase of sepsis or prophylactic effects in patients. Also frequency of sepsis could be studied in cohort of patients receiving infliximab.

\section{Acknowledgements}

Alper B. Iskit has been supported by the Turkish Academy of Sciences, in the framework of the Young Scientist Award Program (EA-TUBA-GEBIP/2001-2-11). This study received no specific grant from any funding agency in the public, commercial, or notfor-profit sectors.

\section{Conflict of interest}

The authors declare that they have no conflict of interest. 


\section{References}

Altintas, N., Erboga, M., Aktas, C., Bilir, B., Aydin, M., Sengul, A., et al. 2016. Protective Effect of Infliximab, a Tumor Necrosis Factor-Alfa Inhibitor, on Bleomycin-Induced Lung Fibrosis in Rats. Inflammation, 39(1): 65-78. doi: 10.1007/s10753-015-0224-z.

Balzan, S., de Almeida Quadros, C., de Cleva, R., Zilberstein, B., and Cecconello, I. 2007. Bacterial translocation: overview of mechanisms and clinical impact. J. Gastroenterol. Hepatol. 22(4): 464-471. doi: $10.1111 / \mathrm{j} .1440-1746.2007 .04933 . x$.

De Backer, D., Orbegozo Cortes, D., Donadello, K., and Vincent, J.-L. 2014. Pathophysiology of microcirculatory dysfunction and the pathogenesis of septic shock. Virulence, 5(1): 73-79. doi: 10.4161/viru.26482.

Dellinger, R.P., Levy, M.M., Rhodes, A., Annane, D., Gerlach, H., Opal, S.M., et al. 2013. Surviving Sepsis Campaign: international guidelines for management of severe sepsis and septic shock, 2012. Intensive Care Med. 39(2): 165-228. doi: 10.1007/s00134-012-2769-8.

Duran-Bedolla, J., Montes de Oca-Sandoval, M.A., Saldana-Navor, V., Villalobos-Silva, J.A., Rodriguez, M.C., and Rivas-Arancibia, S. 2014. Sepsis, mitochondrial failure and multiple organ dysfunction. Clin. Invest. Med. 37(2): E58-69. Available from http://cimonline.ca/index.php/cim/article/view/21087.

Erdem, A., Sevgili, A.M., Akbiyik, F., Atilla, P., Cakar, N., Balkanci, Z.D., et al. 2007. Tezosentan attenuates organ injury and mesenteric blood flow decrease in endotoxemia and cecal ligation and puncture. J. Surg. Res. 141(2): 211-219. doi: 10.1016/j.jss.2006.08.028.

Fei, Y., Wang, W., Kwiecinski, J., Josefsson, E., Pullerits, R., Jonsson, I.M., et al. 2011. The combination of a tumor necrosis factor inhibitor and antibiotic alleviates staphylococcal arthritis and sepsis in mice. J. Infect. Dis. 204(3): 348-357. doi: 10.1093/infdis/jir266.

Ferro, T., Neumann, P., Gertzberg, N., Clements, R., and Johnson, A. 2000. Protein kinase C-alpha mediates endothelial barrier dysfunction induced by TNF-alpha. Am. J. Physiol. Lung. Cell. Mol. Physiol. 278(6): L1107-1117. Available from http://ajplung.physiology.org/content/278/6/L1107.long.

Guo, Z., Wang, S., Jiao, Q., Xu, M., and Xu, Z. 2009. Soluble TNFR II/IgG1 Fc fusion protein treatment in the LPS-mediated septic shock of rats. Biomed. Pharmacother. 63(7): 537-542. doi: 10.1016/j.biopha.2008.08.012.

Hubbard, W.J., Choudhry, M., Schwacha, M.G., Kerby, J.D., Rue, L.W., 3rd, Bland, K.I., et al. 2005. Cecal ligation and puncture. Shock, 24 Suppl 1: 52-57. doi: 10.1097/01.shk.0000191414.94461.7e.

Iskander, K.N., Osuchowski, M.F., Stearns-Kurosawa, D.J., Kurosawa, S., Stepien, D., Valentine, C., et al. 2013. Sepsis: multiple abnormalities, heterogeneous responses, and evolving understanding. Physiol. Rev. 93(3): 1247-1288. doi: 10.1152/physrev.00037.2012.

Kalea, A.Z., Clark, K., Schuschke, D.A., Kristo, A.S., and Klimis-Zacas, D.J. 2010. Dietary enrichment with wild blueberries (Vaccinium angustifolium) affects the vascular reactivity in the aorta of young spontaneously hypertensive rats. J. Nutr. Biochem. 21(1): 14-22. doi: 10.1016/j.jnutbio.2008.09.005.

Kawabe, T., Harris, P.D., Zakaria, E.L., and Garrison, R.N. 2003. Sepsis alters vessel contraction by adrenoceptor-induced nitric oxide and prostanoid. J. Surg. Res. 110(2): 352-359. doi: 10.1016/S00224804(03)00029-5.

Koh, I.H., Menchaca-Diaz, J.L., Farsky, S.H., Siqueira, A.F., Ruiz-Silva, M., Juliana, P., et al. 2002. Injuries to the mesenteric microcirculation due to bacterial translocation. Transplant. Proc. 34(3): 1003-1004. doi: 10.1016/S0041-1345(02)02757-4. 
Lee, J.H., Cho, J.H., Yeo, J., Lee, S.H., Yang, S.H., Sung, Y.C., et al. 2013. The pharmacology study of a new recombinant TNF receptor-hyFc fusion protein. Biologicals, 41(2): 77-83. doi: 10.1016/j.biologicals.2012.09.001.

Ley, K., Laudanna, C., Cybulsky, M.I., and Nourshargh, S. 2007. Getting to the site of inflammation: the leukocyte adhesion cascade updated. Nat. Rev. Immunol. 7(9): 678-689. doi: 10.1038/nri2156.

Lis, K., Kuzawinska, O., and Balkowiec-Iskra, E. 2014. Tumor necrosis factor inhibitors - state of knowledge. Arch. Med. Sci. 10(6): 1175-1185. doi: 10.5114/aoms.2014.47827.

Marik, P.E. 2011. Surviving sepsis: going beyond the guidelines. Ann. Intensive Care, 1(1): 17. doi: 10.1186/2110-5820-1-17.

Ozer, E.K., Gunduz, M.G., El-Khouly, A., Sara, M.Y., Simsek, R., Iskit, A.B., et al. 2015. Microwaveassisted synthesis of condensed 1,4-dihydropyridines as potential calcium channel modulators. Turk. J. Chem. 39(4): 886-896. doi: 10.3906/kim-1412-72.

Ozer, E.K., and Iskit, A.B. 2016. Effects of endothelin and nitric oxide on cardiac muscle functions in experimental septic shock model. Hum. Exp. Toxicol. 35(3): 267-275. doi: 10.1177/0960327115583363.

Pergel, A., Kanter, M., Yucel, A.F., Aydin, I., Erboga, M., and Guzel, A. 2012. Anti-inflammatory and antioxidant effects of infliximab in a rat model of intestinal ischemia/reperfusion injury. Toxicol. Ind. Health, 28(10): 923-932. doi: 10.1177/0748233711427056.

Qiu, P., Cui, X., Barochia, A., Li, Y., Natanson, C., and Eichacker, P.Q. 2011. The evolving experience with therapeutic TNF inhibition in sepsis: considering the potential influence of risk of death. Expert Opin. Investig. Drugs, 20(11): 1555-1564. doi: 10.1517/13543784.2011.623125.

Raven, K. 2012. Rodent models of sepsis found shockingly lacking. Nat. Med. 18(7): 998. doi: 10.1038/nm0712-998a.

Saritemur, M., Un, H., Cadirci, E., Karakus, E., Akpinar, E., Halici, Z., et al. 2015. Tnf-alpha inhibition by infliximab as a new target for the prevention of glycerol-contrast-induced nephropathy. Environ. Toxicol. Pharmacol. 39(2): 577-588. doi: 10.1016/j.etap.2015.01.002.

Scallon, B., Cai, A., Solowski, N., Rosenberg, A., Song, X.Y., Shealy, D., et al. 2002. Binding and functional comparisons of two types of tumor necrosis factor antagonists. J. Pharmacol. Exp. Ther. 301(2): 418-426. doi: 10.1124/jpet.301.2.418.

Schulte, W., Bernhagen, J., and Bucala, R. 2013. Cytokines in sepsis: potent immunoregulators and potential therapeutic targets--an updated view. Mediators Inflamm. 2013: 165974. doi: $10.1155 / 2013 / 165974$.

Shimaoka, M., and Park, E.J. 2008. Advances in understanding sepsis. Eur. J. Anaesthesiol. Suppl. 42: 146-153. doi: 10.1017/S0265021507003389.

Singer, M., Deutschman, C.S., Seymour, C.W., Shankar-Hari, M., Annane, D., Bauer, M., et al. 2016. The Third International Consensus Definitions for Sepsis and Septic Shock (Sepsis-3). JAMA. 315(8): 801-810. doi: 10.1001/jama.2016.0287.

Tunez, I., Feijoo, M., Huerta, G., Montilla, P., Munoz, E., Ruiz, A., et al. 2007. The effect of infliximab on oxidative stress in chronic inflammatory joint disease. Curr. Med. Res. Opin. 23(6): 1259-1267. doi: 10.1185/030079907X187955.

Yuksel, B.C., Serdar, S.E., Tuncel, A., Uzum, N., Ataoglu, O., Atan, A., et al. 2009. Effect of tempol, a membrane-permeable radical scavenger, on mesenteric blood flow and organ injury in a murine cecal ligation and puncture model of septic shock. Eur. Surg. Res. 43(2): 219-227. doi: $10.1159 / 000225984$. 
Yuksel, E., Hasanreisoglu, B., Yuksel, N., Yilmaz, G., Ercin, U., and Bilgihan, A. 2014. Comparison of acute effect of systemic versus intravitreal infliximab treatment in an experimental model of endotoxin-induced uveitis. J. Ocul. Pharmacol. Ther. 30(1): 74-80. doi: 10.1089/jop.2012.0238. 


\section{TABLES}

Table 1. The potency and efficacy of phenylephrine for aortic musle contractions that were obtained from dose-response curves in the Fig.3.

\begin{tabular}{lll}
\hline & $-\log$ EC50 (pD2) & Emax \\
\hline Sham & $7.29 \pm 0.28$ & $1.52 \pm 0.41$ \\
CLP & $6.76 \pm 0.23^{* *}$ & $0.85 \pm 0.30^{* * *}$ \\
Sham+infliximab & $7.54 \pm 0.29$ & $1.33 \pm 0.33$ \\
CLP+infliximab & $7.87 \pm 0.40^{* *}, \# \#$ & $1.12 \pm 0.29^{*}, \#$ \\
\hline
\end{tabular}

Data were analyzed with one-way ANOVA followed by Newman-Keuls multiple comparison post-test. $n=6-7$ for the groups. *Denotes significant difference between Sham $v s$. other groups. \#Denotes significant difference between CLP vs. CLP+infliximab group $(*, \#, p<0.05$; $* *, p<0.01 ; * * *$,\#\#\#, $p<0.001)$.

Table 2. Biochemical blood parameters.

\begin{tabular}{llllll}
\hline & AST & ALT & LDH & BUN & Cr \\
\hline Sham & $124 \pm 30$ & $53 \pm 18$ & $397 \pm 171$ & $43 \pm 18$ & $0.49 \pm 0.12$ \\
CLP & $272 \pm 95^{* *}$ & $127 \pm 58^{*}$ & $1117 \pm 410^{* *}$ & $94 \pm 34^{* *}$ & $0.68 \pm 0.24^{*}$ \\
Sham+infliximab & $107 \pm 38$ & $46 \pm 16$ & $393 \pm 180$ & $48 \pm 17$ & $0.52 \pm 0.22$ \\
CLP+infliximab & $157 \pm 40^{\# \#}$ & $67 \pm 51^{\#}$ & $555 \pm 318^{\# \#}$ & $49 \pm 25^{\#}$ & $0.51 \pm 0.11^{\#}$ \\
\hline
\end{tabular}

Data were analyzed with one-way ANOVA followed by Newman-Keuls multiple comparison post-test. $n=6-7$ for the groups. *Denotes significant difference between Sham vs. other groups. \#Denotes significant difference between CLP vs. CLP+infliximab group $(*, \#, p<0.05$; **,\#\#, $p<0.01)$. 
Table 3: Serum cytokine levels.

\begin{tabular}{llll}
\hline & TNF- $\alpha$ & Il-1 & IL-6 \\
\hline Sham & $70 \pm 27$ & $32 \pm 19$ & $21 \pm 14$ \\
CLP & $417 \pm 178^{* *}$ & $340 \pm 235^{*}$ & $544 \pm 388^{* *}$ \\
Sham+infliximab & $67 \pm 29$ & $31 \pm 19$ & $24 \pm 15$ \\
CLP+infliximab & $88 \pm 26^{\# \#}$ & $88 \pm 47^{\#}$ & $41 \pm 19^{\# \#}$ \\
\hline
\end{tabular}

Data were analyzed with one-way ANOVA followed by Newman-Keuls multiple comparison post-test. $n=6-7$ for the groups. *Denotes significant difference between Sham vs. other groups. \#Denotes significant difference between CLP vs. CLP+infliximab group $(*, \#, p<0.05$; **,\#\#, $p<0.01)$.

Table 4: Tissue MDA and GSH levels.

\begin{tabular}{lllllllll}
\hline & Liver & Lung & Spleen & Kidney & Liver & Lung & Spleen \\
& MDA & MDA & MDA & MDA & GSH & GSH & $\begin{array}{l}\text { GSH } \\
\text { GSH }\end{array}$ \\
\hline Sham & $4.58 \pm 1.27$ & $4.32 \pm 1.15$ & $3.02 \pm 0.99$ & $4.84 \pm 1.32$ & $123 \pm 42$ & $13.60 \pm 3.91$ & $7.47 \pm 2.84$ & $13.24 \pm 2.70$ \\
CLP & $8.94 \pm 2.60^{* * *}$ & $7.32 \pm 1.75^{* * *}$ & $6.06 \pm 1.47^{* * *}$ & $7.89 \pm 1.22^{* *}$ & $28 \pm 14^{* *}$ & $6.89 \pm 2.16^{*}$ & $2.77 \pm 1.49^{* *}$ & $7.01 \pm 1.32^{* *}$ \\
Sham+infliximab & $4.77 \pm 1.21$ & $4.80 \pm 1.32$ & $3.28 \pm 1.06$ & $4.84 \pm 1.08$ & $107 \pm 39$ & $12.55 \pm 4.19$ & $7.78 \pm 2.34$ & $12.58 \pm 3.22$ \\
CLP+infliximab & $6.05 \pm 1.56^{\# \#}$ & $4.99 \pm 1.20^{\# \#}$ & $3.06 \pm 0.91^{\# \# \#}$ & $5.55 \pm 1.92^{\# \#}$ & $95 \pm 25^{\# \#}$ & $10.25 \pm 4.36$ & $8.13 \pm 3.36^{\# \#}$ & $10.35 \pm 3.63^{*}, \#$ \\
\hline
\end{tabular}

Data were analyzed with one-way ANOVA followed by Newman-Keuls multiple comparison post-test. $n=6-7$ for the groups. *Denotes significant difference between Sham vs. other groups. \#Denotes significant difference between CLP vs. CLP+infliximab group (*,\#, $p<0.05$; $* *$,\#\#, $p<0.01 ; * * *, \# \# \#, p<0.001)$. 


\section{Figure Captions:}

Fig. 1. Effects of infliximab on sepsis induced mortality. The survival rates were assessed for 96 hours and analysed using the Kaplan-Meier plots and Gehan-Breslow-Wilcoxon tests. $n=7$ 8 for the groups. *Denotes significant difference between Sham vs. other groups. \#Denotes significant difference between CLP $v s$. CLP+infliximab group $(*, p<0.05 ; * * *$, \#\#\#, $p<0.001)$.

Fig. 2. Effects of infliximab on mesenteric arterial blood flow. Data were analyzed with oneway ANOVA followed by Newman-Keuls multiple comparison post-test. $n=6-7$ for the groups. *Denotes significant difference between Sham vs. other groups. \#Denotes significant difference between CLP vs. CLP+infliximab group (***,\#\#\#, $p<0.001)$.

Fig. 3. The comparison of the phenylephrine-induced contractile responses in isolated aortic rings that were recorded as changes in grams of force. Data were analyzed with generalized estimating equations to account for repeated measures ANOVA test followed by NewmanKeuls multiple comparison post-test, as well as contractile responses to each phenylephrine dose were compared with two-way ANOVA followed by Newman-Keuls multiple comparison post-test. $n=6-7$ for the groups. a; significant difference between Sham and CLP group, b; significant difference between CLP and CLP+infliximab group, c; significant difference between Sham and CLP+infliximab, d; significant difference between Sham and Sham+infliximab group (a, $p<0.05$; bb,cc, $p<0.01$; aaa,bbb,ccc, $p<0.001$ ).

Fig.4. Effects of infliximab on sepsis induced histopathological changes. The photographs show the representative histopathological sections of liver (1), lung (2), spleen (3) and kidney (4) obtained from the rats in Sham (1A, 2A, 3A, 4A), Sham+infliximab (1B, 2B, 3B, 4B), CLP (1C, 2C, 3C, 4C) and CLP+infliximab (1D, 2D, 3D, 4D) groups. In the Sham group, the liver (Fig.4-1A), lung (Fig.4-2A), spleen (Fig.4-3A) and kidney (Fig.4-4A) tissues showed normal histopathological structure. There were also normal liver (Fig.4-1B), lung (Fig.4-2B), spleen (Fig.4-3B) and kidney (Fig.4-4B) tissues in the Sham+infliximab group. Fig.4-1C; septic liver tissue with moderate sinusoidal portal hemorrhage (arrowhead) and mild inflammatory mononuclear cell infiltration in the parenchyma and periportal area (arrow). Fig.4-1D; normal liver architecture in CLP+infliximab group. Fig.4-2C; the septic lung tissue with moderate intra-alveolar hemorrhage (arrowhead), hemosiderin-laden macrophages (4point star) and as well as moderate peribronchial and interstitial inflammation that was 
concentrated near the vessel wall (arrow). Fig.4-2D; the reduced lung injury in CLP+infliximab group with little inflammation (arrow). Fig.4-3C; septic spleen tissue with moderate hemorrhage (arrowhead), severe inflammation (arrow), hemosiderin-laden macrophages (4-point star). Fig.4-3D; the reduced spleen injury in CLP+infliximab group with little hemorrhage (arrowhead) and inflammation (arrow). Fig.4-4C; septic kidney tissue with moderate hemorrhage (arrowhead). Fig.4-4D; normal kidney architecture in CLP+infliximab group. 


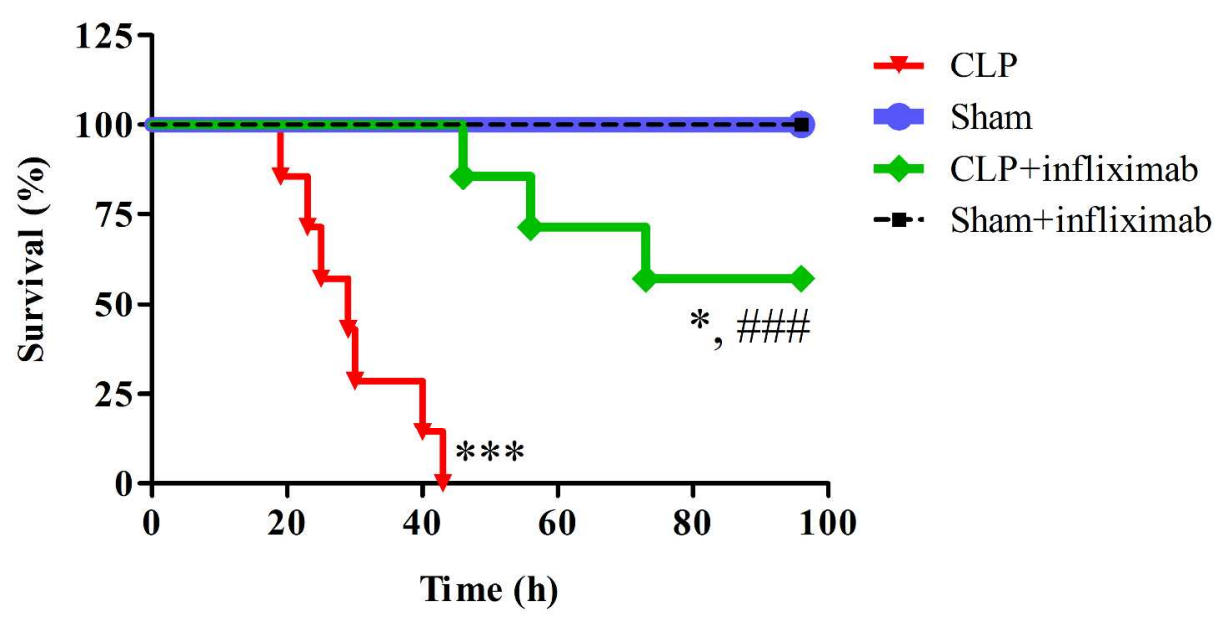

Figure 1

$268 \times 147 \mathrm{~mm}(300 \times 300 \mathrm{DPI})$ 


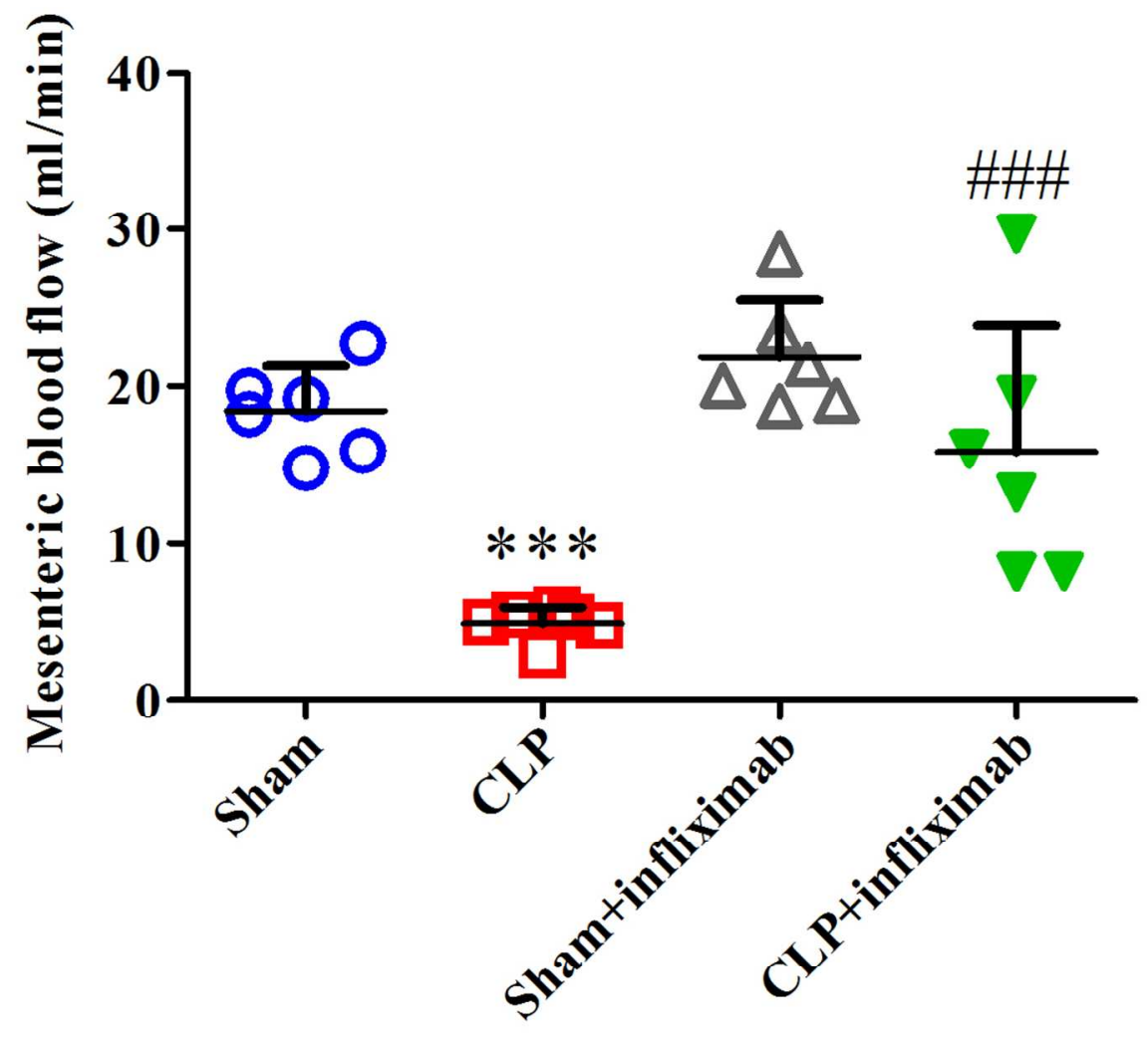

Figure 2

$104 \times 90 \mathrm{~mm}(300 \times 300$ DPI $)$ 


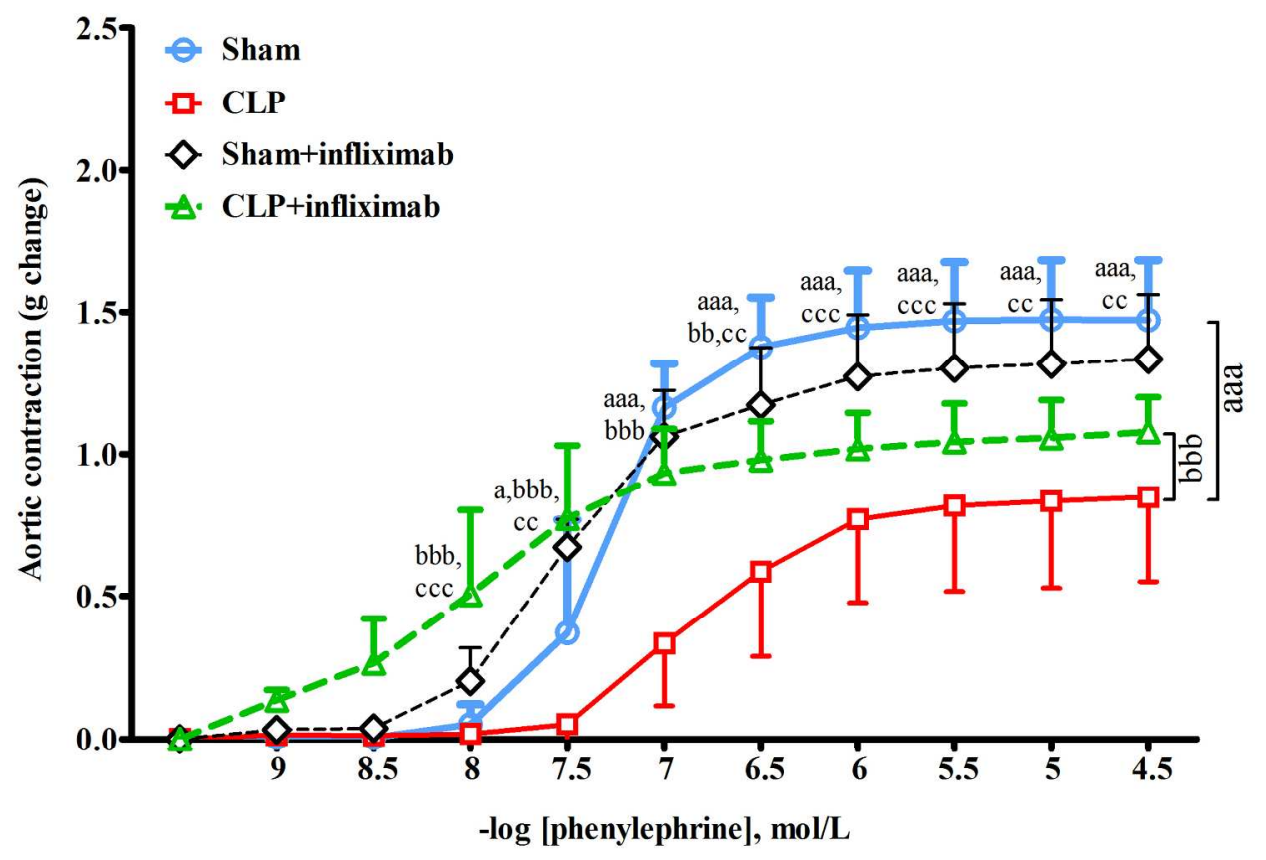

Figure 3

$195 \times 135 \mathrm{~mm}(300 \times 300$ DPI $)$ 


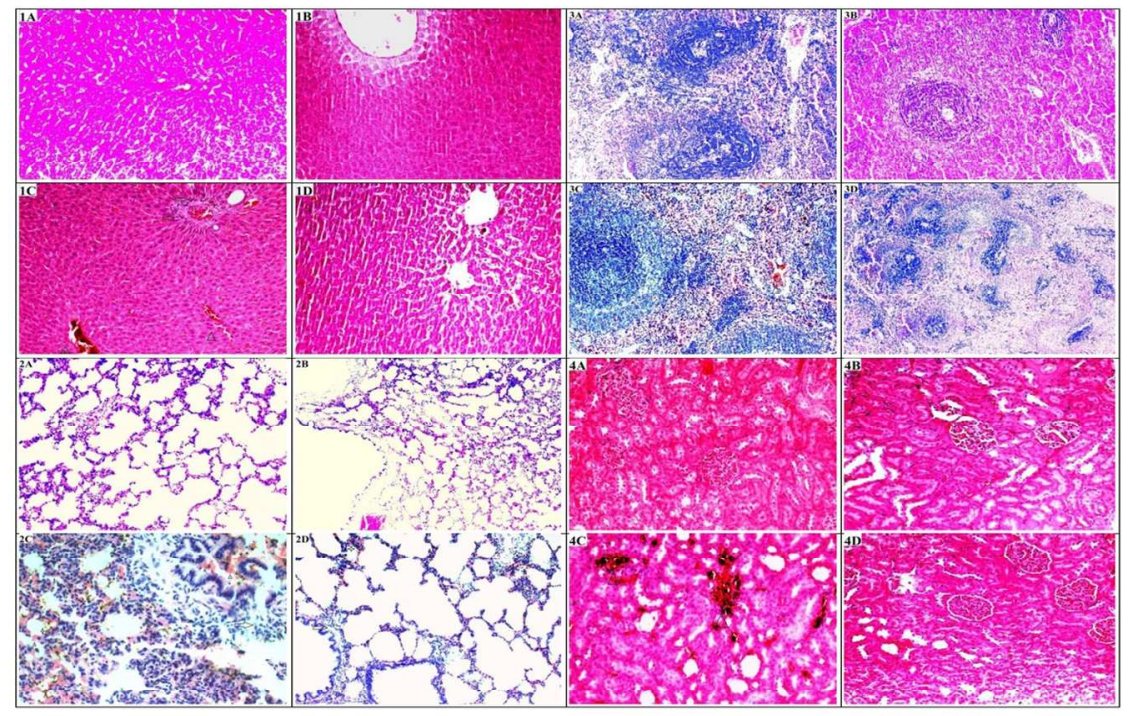

Figure 4

$62 \times 44 \mathrm{~mm}(600 \times 600 \mathrm{DPI})$ 